\title{
/I/IOMENTUM
}

QUARTERLY

ZEITSCHRIFT FÜR

SOZIALEN FORTSCHRITT

2020 I Vol. 9. No. 1, p. $18-34$

\section{NEET-Jugendliche in Österreich: Problemausmaß, volkswirtschaftliche Kosten und Handlungsempfehlungen}

\author{
Johann Bacher
}

Zusammenfassung

Der NEET-Indikator ist zu einem festen Bestandteil der Sozialberichterstattung geworden und dient als Referenzgröße für sozial-, arbeits- und bildungspolitische Maßnahmen. Der Beitrag untersucht die Betroffenheit von NEET (Not in Education, Employment or Training) in Österreich im Jahr 2018 und stell statistische Zusammenhänge dar. Darüber hinaus wird eine Schätzung der volkswirtschaftlichen Kosten von NEET vorgenommen. Aus den bisherigen Forschungen zu NEET werden schließlich Handlungsempfehlungen abgeleitet. Ein kurzes Fazit mit Bezug zum aktuellen Regierungsprogramm der neuen Bundesregierung schließt den Beitrag ab.

Schlagwörter: NEET-Quote, volkswirtschaftliche Kosten, Handlungsempfehlungen

NEET Youth in Austria - Extent, Economic Costs and Recommendations

Abstract

The NEET indicator has become an integral part of social reporting and serves as a benchmark for social, labor and educational policy measures. The article examines the NEET (Not in education, employment or training) risk in Austria in 2018 and presents statistical relationships. In addition, the economic costs of NEET are estimated. Finally, recommendations for action are derived from previous research on NEET. A short conclusion with reference to the current program of the new federal government concludes the contribution.

Keywords: NEET, statistical analysis, economic costs, recommendation

\footnotetext{
"IIIIIIIIIIIIIIIIIIIIIIIIIIIIIIIIIIIIIII

* Johann Bacher, Johannes Kepler Universität Linz, Institut für Soziologie. E-Mail: johann.bacher@jku.at.

Der Autor bedankt sich für die hilfreichen anonymen Gutachten, die u. a. zu wichtigen Ergänzungen geführt haben.
} 
1. Das Konzept der NEET-Jugendlichen als wichtiger sozialpolitischer Indikator

Das Konzept der NEET-Jugendlichen ist seit seiner ersten Rezeption ein fester Bestandteil sozialpolitischer Berichterstattung auf österreichischer und europäischer Ebene (Eurostat 2019; Statistik Austria 2019b; O'Reilly et al. 2019). ${ }^{1}$ Als NEET-Jugendliche (NEET $=$ Not in Education, Employment or Training) werden Angehörige einer bestimmten Altersgruppe betrachtet, die sich zum Erhebungszeitpunkt nicht in (Aus-) Bildung befinden, nicht erwerbstätig sind und auch an keiner Trainingsmaßnahme teilnehmen. Betrachtet wird in der Regel die Altersgruppe der 15- bis 24-Jährigen, mitunter werden aber auch Alterskohorten mit anderen Altersgrenzen untersucht, z. B. 15- bis 29-Jährige (Eurofund 2012: 73; Bălan 2015). ${ }^{2}$ Eurostat (2019) ermöglicht sogar die Berechnung von NEET-Quoten für unterschiedliche Altersgruppen von 15 bis 34 Jahren.

In der Fachliteratur (zusammenfassend Bacher et al. 2014) wird das NEET-Konzept als sinnvolle Ergänzung zur Erfassung der Jugendarbeitslosigkeit gesehen, da auch Jugendliche bzw. junge Erwachsene berücksichtigt werden, die aus unterschiedlichen Gründen keine Erwerbstätigkeit suchen. Gründe hierfür können beispielsweise sein, dass sich die NEET-Jugendlichen wegen erfolgloser Suche aus dem Arbeitsmarkt zurückgezogen haben oder wegen Erkrankungen nicht umfangreich erwerbstätig sein können. Der genannte Vorteil gegenüber dem Konzept der Jugendarbeitslosigkeit ist mit dem Nachteil verbunden, dass die Gruppe der NEET-Jugendlichen heterogen ist (z. B. European Commission 2018) und sowohl Jugendliche mit einem sehr hohen Ausgrenzungsrisiko erfasst, wie z. B. NEET-Jugendliche mit dauerhaften psychischen Beeinträchtigungen, als auch Jugendliche, die sich

1 Das Konzept wurde in Großbritannien in den 1980er-Jahren entwickelt zur Erfassung jener „nicht-registrierten" Jugendlichen, die mit keiner der üblichen statistischen Erhebungskategorien (z. B. ,erwerbstätig“, „arbeitslos“ etc.) erfasst werden. Zur Kennzeichnung dieser Jugendlichen wurde ursprünglich die Bezeichnung "Zone o/Zone Zero" (später Zone A) verwendet. Anfang der 1990er-Jahre wurde diese stigmatisierende Begrifflichkeit durch den Ausdruck NEET ersetzt. Mit der Jahrtausendwende verbreitete sich der Begriff rapide, nicht nur in Europa, sondern auch in anderen Ländern (zur Geschichte siehe Mascherini (2018)).

2 Wegen der Altersspanne wäre es daher zutreffender von Jugendlichen und jungen Erwachsenen zu sprechen. Aus Gründen der sprachlichen Vereinfachung soll die Bezeichnung NEET-Jugendliche beibehalten werden. nach erfolgreicher Matura in den Sommermonaten in einer Warteposition für ein weiterführendes Studium befinden. Dieses methodische Problem wird dadurch $\mathrm{zu}$ lösen versucht, dass Subgruppen von Jugendlichen betrachtet werden. Bacher et al. (2014) beispielsweise konnten sieben Gruppen von NEET-Jugendlichen mittels einer latenten Klassenanalyse ermitteln.

Weiterführende Analysen (Bacher et al. 2016) zeigen dabei, dass dauerhafte psychische Beeinträchtigungen bei der Verursachung von NEET eine wichtige Rolle spielen. $20,5 \%$ der sogenannten inaktiven NEET-Jugendlichen, das sind jene NEET-Jugendliche, die zum Erhebungszeitpunkt aktiv keine Arbeitsstelle suchen oder diese zwar suchen, aber nicht unmittelbar dem Arbeitsmarkt zur Verfügung stehen, berichten von dauerhaften psychischen Beeinträchtigungen, unter den aktiven NEET-Jugendlichen, die zum Erhebungszeitpunkt eine Arbeitsstelle suchen, sind dies 8,7 \% (Bacher et al. 2016: 42).

Neben der Heterogenität wird an dem NEETKonzept dahingehend Kritik geübt, dass die Gefahr der Stigmatisierung der von NEET betroffenen Jugendlichen besteht (z. B. Gracey/Kelly 2010).

Trotz dieser kritischen Punkte ist festzuhalten, dass der NEET-Indikator ein sinnvolles sozialpolitisches Instrument ist, wobei bei der konkreten Ausformulierung von sozialpolitischen Maßnahmen die Heterogenität der erfassten Gruppen zu berücksichtigen ist und Stigmatisierungen vermieden werden sollten (European Commission 2018; Bacher et al. 2014). Der NEET-Status beeinträchtigt die Lebenschancen der betroffenen Jugendlichen unmittelbar und langfristig (z. B. Bonner 2017; Coles et al. 2002). Er verursacht auch für die Gesellschaft beträchtliche soziale, politische und wirtschaftliche Folgekosten (Eurofund 2012).

Aufbauend auf diesen Grundüberlegungen soll folgenden Fragen nachgegangen werden:

- Wie viele Jugendliche und junge Erwachsene sind von einer NEET-Situation in Österreich betroffen? Wie sieht die Situation in den Bundesländern aus?

- Wer ist wie stark betroffen? Gibt es Unterschiede nach soziodemografischen Merkmalen? Bestehen Unterschiede nach Bundesländern?

- Welche volkswirtschaftlichen Kosten verursacht NEET?

- Welche Empfehlungen für Maßnahmen lassen sich aus den Erkenntnissen ableiten?

Ziel des Beitrags ist, über die aktuelle Situation in Österreich zu informieren, um vorhandene Literaturreviews (z. B. Litschel 2019) zu ergänzen. Der Beitrag 
ist wie folgt aufgebaut. Kapitel 2 beschreibt das methodische Vorgehen, mit dem die in den folgenden beiden Kapiteln berichteten empirischen Befunde ermittelt wurden. Kapitel 3 referiert über die Betroffenheit von NEET und stellt statistische Zusammenhänge dar. Kapitel 4 nimmt eine Schätzung der volkswirtschaftlichen Kosten von NEET vor. Aus den bisherigen Forschungen zu NEET werden in Kapitel 5 Handlungsempfehlungen abgeleitet. Kapitel 6 zieht ein kurzes Fazit mit Bezug zum aktuellen Regierungsprogramm der seit Jänner 2020 amtierenden türkis-grünen Bundesregierung.

\section{Methodisches Vorgehen}

Zur Schätzung der NEET-Quote wird auf den Mikrozensus (MZ) zurückgegriffen, konkret auf das Jahr 2018. Auch Statistik Austria und Eurostat verwenden diese Datenquelle. Der MZ ist Teil des International Labour Force Survey. Es handelt sich um eine Haushaltsstichprobe (Haslinger/Kytir 2006). Ausgewählt werden mittels eines geschichteten Zufallsstichprobenverfahrens Haushalte. Erhoben werden Haushaltsmerkmale und Personenmerkmale für jede im Haushalt lebende Person. Der MZ wird in Österreich viermal pro Jahr mit einer Fünftelrotation durchgeführt. Erfasst werden jeweils ca. 20.000 Haushalte pro Quartal, in denen 44.00o Personen leben. Ein Fünftel der befragten Haushalte und der darin lebenden Personen scheidet quartalsmäßig aus der Befragung aus und wird durch ein neues Fünftel ersetzt.

In die Berechnung werden die 15- bis 24-Jährigen einbezogen. Absolut betrachtet sind das $n=18.462$ Personen. Bei der Berechnung der NEET-Quote wird das von der Statistik Austria und Eurostat verwendete Vorgehen eingesetzt, das aus folgenden Schritten besteht: ${ }^{3}$

1. Zivil- und Präsenzdiener werden aus der Berechnung ausgeschlossen. Sie gehen also nicht in den Nenner ein. Die Zahl der 15- bis 24-Jährigen reduziert sich damit auf $\mathrm{n}=18.077$.

2. Als Nicht-NEET-Jugendliche werden Jugendliche bezeichnet, für die eines der folgenden Kriterien zutrifft:

a. Teilhabe im Bildungssystem (Schulbesuch oder Studium) im Referenzzeitraum;

3 Ich bedanke mich an dieser Stelle bei Daniela Gumprecht von der Statistik Austria für die Erörterungen zur Berechnung des NEET-Status. b. Erwerbstätigkeit im Umfang von mindestens einer Stunde im Referenzzeitraum;

c. Teilnahme an einer beruflichen Weiterbildung und/oder Besuch eines Freizeitkurses ${ }^{4}$ im Ausmaß von mindestens einer Stunde im Referenzzeitraum.

Referenzzeitraum ist jeweils eine Woche, die bei der Befragung vorgegeben wird. Dabei soll es sich im Idealfall um die der Befragungswoche vorausgehende Woche handeln. Zwischen Referenzwoche und Befragungswoche darf - außer im Sommerquartal - maximal ein Zeitraum von fünf Wochen liegen (Baumgartner et al. 2017).

Statistisch betrachtet handelt es sich beim MZ um ein komplexes Stichprobendesign (Bacher 2009). Durch die wiederholte Befragung von Personen in einem Haushalt entsteht ein Klumpeneffekt, der bei der Durchführung von statistischen Tests und der Berechnung von statistischen Signifikanzen berücksichtigt werden muss. In dieser Studie wird der in Bacher und Tamesberger (2011) ermittelte Designeffekt für den NEET-Status von 5,39 verwendet. Die effektive Stichprobengröße für statistische Signifikanztests liegt damit bei neff=3.354 und ist im Vergleich zu Stichprobengrößen repräsentativer Umfragen mit einer Fallzahl von $\mathrm{n}=\mathbf{1 . 0 0 0}$ oder $\mathrm{n}=\mathbf{2} .000$ Personen noch immer groß.

Eine größere Datenbasis könnte gewonnen werden, wenn bei der Analyse des NEET-Status auf sogenannte Registerdaten zurückgegriffen werden könnte, wie dies etwa bei den frühen SchulabgängerInnen (Steiner et al. 2016) der Fall ist, wo Daten aus dem „Bildungsbezogenen Erwerbskarrierenmonitoring" (BibEr) verwendet werden konnten. Registerdaten zur Analyse des Zusammenhangs von NEET-Status und Gesundheitszustand nutzen Pruckner und Schober (2016). Gut abgebildet werden konnten aktive NEET-Jugendliche, weniger gut inaktive NEET-Jugendliche. Es wäre grundsätzlich wünschenswert, dass in Zukunft auch zur Analyse des NEET-Status Registerdaten zur Verfügung stehen würden. Registerdaten würden sich wegen der großen Fallzahl vor allem eignen, um Risiken von kleinen Gruppen oder kleinräumig (z. B. auf Bezirksebene) $\mathrm{zu}$ schätzen. Auch Kausalanalysen würden erleich-

4 In vorausgehenden Studien des Autors wurden Freizeitkurse nicht berücksichtigt. Präsenz- und Zivildiener wurden dagegen als NEET-Jugendliche betrachtet. Dadurch ergaben sich leicht abweichende Zahlen zu jenen der Statistik Austria. 
Übersicht 1: Vor- und Nachteile von Registerdaten im Vergleich zu Befragungsdaten auf Stichprobenbasis

\begin{tabular}{|c|c|c|}
\hline & Registerdaten & Befragungsdaten auf Stichprobenbasis \\
\hline $\begin{array}{l}\text { Schätzungen für } \\
\text { Gesamtpopulation }\end{array}$ & $\begin{array}{l}\text { Ja, Verzerrungen nicht ausgeschlossen (s.u.), Erfassung } \\
\text { des Phänomens kann Probleme bereiten, da Daten für } \\
\text { andere Zwecke erhoben wurden. }\end{array}$ & $\begin{array}{l}\text { Ja, Verzerrungen nicht ausgeschlossen (s.u.), Erfas- } \\
\text { sung des Phänomens leichter, wenn Primärerhebung } \\
\text { vorliegt, bei MZ nicht der Fall, da Sekundärdaten }\end{array}$ \\
\hline $\begin{array}{l}\text { Kleinräumige Analysen und } \\
\text { Analysen von Subgruppen }\end{array}$ & $\begin{array}{l}\text { Ja, da große Fallzahl, i.d.R. Vollerhebung, Verzerrungen } \\
\text { durch Registrierungsunterschiede möglich. }\end{array}$ & $\begin{array}{l}\text { Nein, da i.d.R. Stichprobe mit kleinerer Fallzahl, falls } \\
\text { möglich, Verzerrungen möglich. }\end{array}$ \\
\hline $\begin{array}{l}\text { Internationale } \\
\text { Vergleichbarkeit }\end{array}$ & $\begin{array}{l}\text { Nein, da häufig nationale Unterschiede bei der Regist- } \\
\text { rierung. }\end{array}$ & Ja, da einheitliches Vorgehen möglich. \\
\hline Verzerrungen & $\begin{array}{l}\text { Möglich durch Registrierung (Filter 1: Person meldet sich } \\
\text { nicht zur Registrierung, Filter 2: Registrierung wird nicht } \\
\text { vorgenommen). }\end{array}$ & $\begin{array}{l}\text { Möglich durch Ausfälle bei der Feldphase und durch } \\
\text { falsche Antworten (z. B. durch soziale Wünschbarkeit). }\end{array}$ \\
\hline Kausalanalysen & $\begin{array}{l}\text { Werden erleichtert, da Längsschnittcharakter verfügbar, } \\
\text { leichtere nachträgliche Randomisierung und leichteres } \\
\text { Auffinden von statistischen Zwillingen zur Wirkungsab- } \\
\text { schätzung wegen großer Fallzahl. } \\
\text { Werden erschwert, da keine intervenierenden Variablen } \\
\text { wie Einstellungen, Werthaltungen und Handlungsgründe } \\
\text { erfragt werden können. }\end{array}$ & $\begin{array}{l}\text { Werden erschwert, da oft nur Querschnittsdaten vorlie- } \\
\text { gen und kleine Fallzahl das Auffinden von statistischen } \\
\text { Zwillingen erschwert. } \\
\text { Werden erleichtert, da intervenierende Variablen, wie } \\
\text { Einstellungen, Werthaltungen und Handlungsgründe, } \\
\text { erfragt werden können (im MZ aber nur begrenzt). }\end{array}$ \\
\hline $\begin{array}{l}\text { Aussagekraft statistischer Signi- } \\
\text { fikanz hinsichtlich Relevanz }\end{array}$ & $\begin{array}{l}\text { Geringer, da bei großen Fallzahlen kleinste Unterschiede } \\
\text { signifikant werden. }\end{array}$ & Größer, da kleinere Fallzahlen vorliegen. \\
\hline
\end{tabular}

tert (siehe Übersicht $1^{5}$ ). Allerdings müsste dazu eine verbindliche Operationalisierung gefunden werden, die sich vermutlich - wie die nationale Definition der Arbeitslosigkeit - von der internationalen Definition des NEET-Status unterscheidet. So könnte es beispielsweise sein, dass bei der nationalen Definition eines NEET-Status erst dann von einer Erwerbstätigkeit gesprochen wird, wenn diese ein bestimmtes Stundenausmaß, z. B. mindestens zwölf Stunden, umfasst, während derzeit für eine Nicht-NEET-Situation eine Stunde Erwerbstätigkeit ausreicht. Für international vergleichende Studien wäre wegen Unterschieden in der Operationalisierung daher nach wie vor eine Verwendung des MZ erforderlich. Geklärt werden müsste auch, wie stark bei Registerdaten vorhandene Filter wirksam sind. Diese bestehen darin, dass sich (1.) Personen zur Registrierung melden müssen und dass (2.) ihre Registrierung auch durchgeführt wird. So kann es z. B. sein, dass sich NEET-Jugendliche gar nicht beim AMS melden, da sie keine Unterstützung und Leistungen erwarten. Oder eine Registrierung als „arbeitslos“ wird abgelehnt, weil bestimmte Voraussetzungen nicht erfüllt sind. Wie stark diese Filter wirken und die Daten verzerren, ist derzeit im Detail nicht bekannt. Die Überschneidung zwischen AMS-Registrierung

5 Siehe dazu die Diskussion in der Versorgungsforschung (Benchimol et al. 2016; Lefering 2016) sowie zu Vorteilen der Registerforschung (Cuaresma et al. 2020). und internationaler Definition von Arbeitslosigkeit, die in die derzeitige NEET-Definition einfließt, ist gering. Nur 52,8 \% der aktiven NEET-Jugendlichen, die entsprechend ihren Angaben arbeitssuchend sind und dem Arbeitsmarkt zur Verfügung stehen, sind 2018 auch beim AMS als arbeitssuchend registriert. ${ }^{6}$ Ein weiterer Vorteil von Nicht-Registerdaten, der aber für den MZ wegen der gesetzlichen Vorgaben weniger greift, ist das Erfassen von Einstellungen, Werthaltungen und Gründen des Handelns in Umfragen. Diese intervenierenden Variablen fehlen im Regelfall bei Registerdaten. Umgekehrt zu den erörterten Verzerrungen von Registerdaten sind natürlich auch Verzerrungen im MZ zu sehen, die dadurch entstehen, dass Befragte z. B. eine geringfügige Erwerbstätigkeit nennen, obwohl sie arbeitslos sind, und damit als Nicht-NEET-Jugendliche gelten, da Erwerbstätigkeit sozial erwünschter ist als Arbeitslosigkeit.

Zusätzlich zum NEET-Status werden noch soziodemografische Variablen betrachtet, deren Verteilung in Tabelle A1 im Anhang angeführt ist. Sie dienen als unabhängige Variablen zur Analyse des NEETStatus. Neben bivariaten Analysen werden explorative Pfadanalysen (Holm 1977) gerechnet, wobei Pfadkoeffizienten mit einem Absolutbetrag größer 0,08 als bedeutsam betrachtet werden. Dieser im Vergleich

6 Diese Analyse ist möglich, da der MZ mit Registerdaten des AMS verknüpft werden kann. 
Tabelle 1: Volkswirtschaftliche Kosten von NEET pro NEET-Jugendlichen nach Eurofund (2012)

\begin{tabular}{lcc}
\hline & 2008 in Euro & 2018 in Euro ${ }^{a)}$ \\
\hline Einkommen aus Transferzahlungen (.,unit public finance cost") & $1.129,-$ & $1.271,-$ \\
$\begin{array}{l}\text { Entgangenes Einkommen inkl. entgangener Einnahmen von Steuern und Sozial- } \\
\text { beiträgen (,.unit resource cost") }\end{array}$ & $14.672,-$ & $16.520,-$ \\
Gesamtkosten (,unit total cost") & $15.801,-$ & $17.791,-$ \\
\hline
\end{tabular}

Quellen: Eurofund (2012), eigene Berechnungen für 2018

a) Es wurde eine Steigerung der Einkommen um 12,6 \% angenommen. Dies entspricht dem geometrischen Mittel aus der Steigerung der Einkommen des 1. Quartals und des Medians (Statistik Austria 2019a).

zum oft verwendeten Schwellenwert von o,10 geringere Wert lässt sich damit begründen, dass dichotome Variablen untersucht werden und bei diesen die bivariaten Korrelationen als Basis der Pfadanalyse numerisch geringer ausfallen. Nur unter sehr restriktiven, empirisch im Regelfall nicht erfüllten Bedingungen wird ein Absolutwert von 1,0 erreicht (Bortz 1999: 219-220). ${ }^{\text {? }}$

Aktualisiert wird in dieser Arbeit auch eine von Eurofund (2012) vorgenommene Schätzung der durch NEET verursachten ökonomischen und sozialen Kosten. Als soziale Kosten betrachten die AutorInnen der Eurofund-Studie ein geringeres Vertrauen in Institutionen sowie eine geringe soziale und politische Teilhabe (zur politischen Partizipation siehe auch Sailer/Tamesberger 2013). Bei den ökonomischen Kosten werden Mindereinnahmen aus entgangenem Erwerbseinkommen und öffentliche Transferleistungen berücksichtigt, wobei NEET-Jugendliche statistisch vergleichbaren erwerbstätigen Jugendlichen gegenübergestellt werden, also Jugendlichen, die sich hinsichtlich des Geschlechts, des Alters, der Bildung usw. nicht von den NEET-Jugendlichen unterscheiden. Der einzige Unterschied besteht darin, dass der/die eine Jugendliche einen NEET-Status hat und der/die andere Jugendliche nicht.

Für die Berechnung ausgewählt wurde von Eurofund die Altersgruppe der 16- bis 29-Jährigen, wobei nur jene NEET-Jugendliche in die Kostenschätzungen einbezogen wurden, die sechs Monate oder länger arbeitslos waren oder die zu den Nichterwerbspersonen zählen. Nicht berücksichtigt wurden somit NEETJugendliche mit einer Arbeitslosigkeitsdauer unter sechs Monaten sowie arbeitslose Jugendliche in Schulungsmaßnahmen, die per Definition nicht als NEETJugendliche gelten. Für das Berechnungsjahr 2008

7 Ein positiver Wert von 1,o beispielsweise kann nur erreicht werden, wenn die Randhäufigkeiten beider Variablen gleich sind. werden für Österreich die in der Tabelle 1 angeführten Werte berichtet (Eurofund 2012: 76). Die Ergebnisse für das entgangene Erwerbseinkommen erscheinen durchaus realistisch. Der ausgewiesene Wert von $€ 14.672$,- liegt knapp über dem 1. Quartil der BruttoErwerbseinkommen von $€$ 11.375,- und damit deutlich unter dem Medianeinkommen von $€$ 24.253,- des Jahres 2008 (Statistik Austria 2019a), was angesichts der geringeren Bildung der NEET-Jugendlichen und der entsprechenden Vergleichsgruppe zu erwarten ist. Die angeführten, relativ geringen Transferzahlungen (z. B. Pensionen, Familienbeihilfe, Wohnbeihilfe etc.) in der Höhe von $€ 1.271$,- sind ebenfalls realistisch, wenn berücksichtigt wird, dass NEET-Jugendliche noch bei ihren (nicht-anspruchsberechtigten) Eltern oder in Partnerschaft leben können und wegen fehlender Ansprüche keine Leistungen aus den sozialen Sicherungssystemen erhalten.

Nicht berücksichtigt werden in den vorgenommenen Berechnungen Kosten, die durch die Inanspruchnahme von sozialen und gesundheitlichen Dienstleistungen oder durch die Teilnahme an Kursen entstehen. Das führt dazu, dass die vorgenommenen Berechnungen die Kosten unterschätzen (Eurofund 2012) und als konservativ bezeichnet werden können.

In Tabelle 1 wurden die Berechnungswerte von Eurofund (2012) für das Jahr 2018 unter Berücksichtigung der Steigerungen der Einkommen aus unselbständiger Tätigkeit (brutto) fortgeschrieben. Es resultieren Gesamtkosten in der Höhe von $€ 17.791$,- pro Jugendlichen. Dieser Wert wird zur Berechnung der volkswirtschaftlichen Kosten von NEET verwendet.

Zusätzlich wird in einer weiteren Modellrechnung der Einnahmeausfall der öffentlichen Hand ermittelt. Dabei wird von einem Bruttojahreseinkommen von $€$ 16.520,- ausgegangen, das erreicht werden könnte, wenn der/die NEET-Jugendliche in den Arbeitsmarkt integriert wird. In diesem Fall entstehen öffentliche Einnahmen aus den Arbeitnehmerbeiträgen 
Abbildung 1: NEET-Quoten 2018 nach Bundesländern

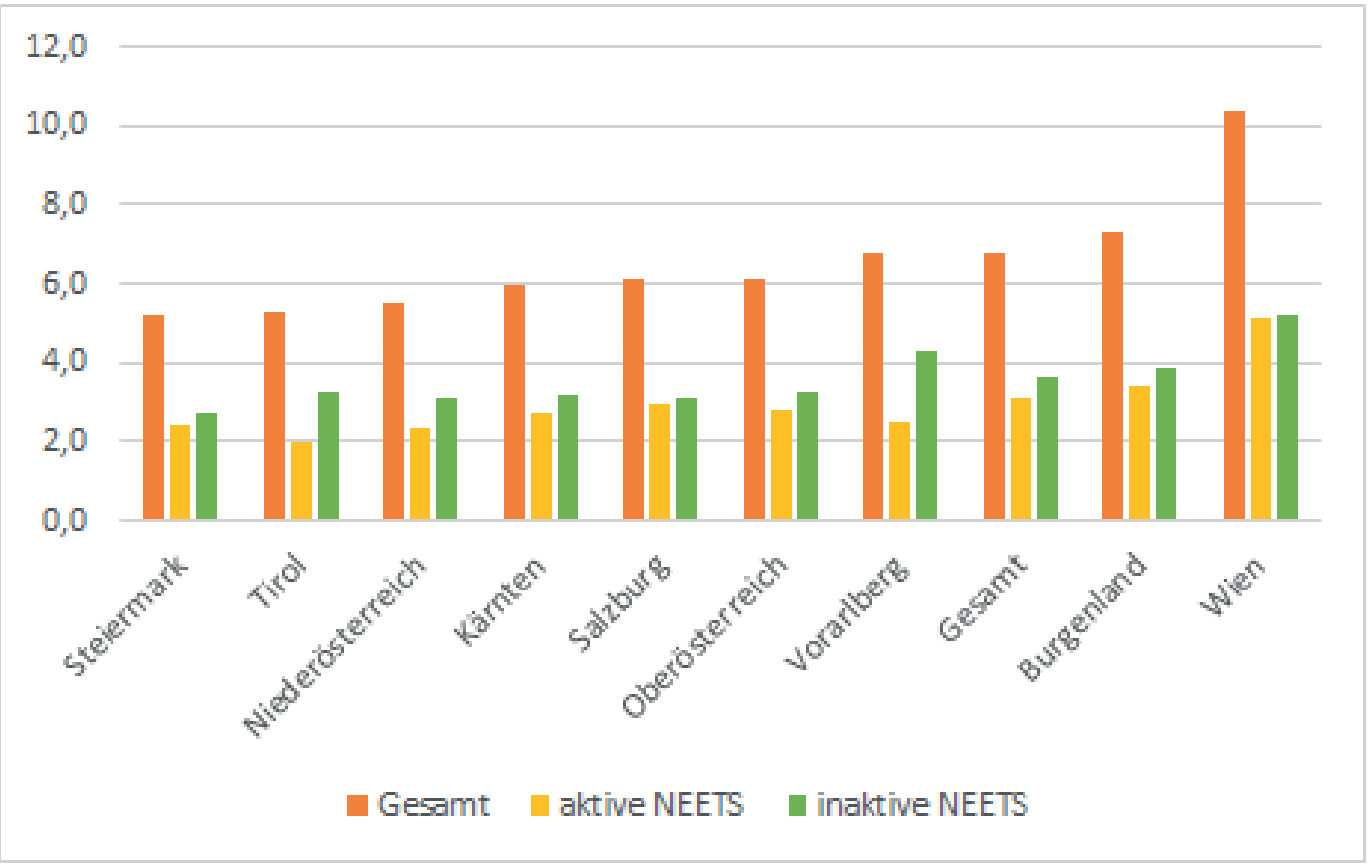

Quelle: Mikrozensus 2018, eigene Berechnungen, $\mathrm{n}_{\mathrm{eff}}=3.354$

zur Lohnsteuer und zur Sozialversicherung, aus den korrespondierenden Dienstgeberabgaben und aus der Umsatzsteuer für den Konsum. Die bei einem Bruttojahreseinkommen anfallenden öffentlichen Einnahmen ${ }^{8}$ belaufen sich auf: Lohnsteuer $€ 84,40$, Sozialversicherungsbeitrag $€ 2.474,28$, Dienstgeberabgaben $€$ 4.635,22 und Umsatzsteuer $€ 2.189,13$. Bezüglich der Umsatzsteuer wurde angenommen, dass $2 \%$ des Nettojahreseinkommens von $€ 13.961,32$ gespart werden und für den restlichen Betrag ein Mischsteuersatz von $16 \%$ für die Umsatzsteuer entrichtet wird. Es wurde also eine Konsumneigung von $98 \%$ angenommen, wie sie Ederer (2017) für das 2. Terzil berichtet. Der durchschnittliche Mehrwertsteuersatz wurde auf der Grundlage der von der Statistik Austria veröffentlichten Umsatzsteuerstatistik 2016 (Statistik Austria 2019c: 36) berechnet.

\section{Betroffenheit von NEET}

Die Anwendung der in Kapitel 2 dargestellten Berechnungsmethode ergibt für 2018 eine NEET-Quote von 6,8 \% unter den 15- bis 24-Jährigen. In Absolutzahlen

8 Die Berechnung erfolgte mittels des Brutto-Netto-Rechner des Bundesministeriums für Finanzen, https://rechner.cpulohn.at/bmf.gv.at/ familienbonusplus/\#bruttoNetto_familienbonus [1.03.2020]. ausgedrückt befanden sich ca. 64.00o Jugendliche in einer NEET-Situation. Im langfristigen Trend lässt sich in Österreich ein Rückgang der NEET-Quote beobachten. Die NEET-Quote lag beispielsweise 2004 bei 9,1 \% (Statistik Austria 2019b). Im Vergleich zu 2017 kam es aber im Jahr 2018 zu einem leichten Anstieg von 6,5 \% auf 6,8 \% (Statistik Austria 2019b), der sich 2019 fortsetzte. Laut rezenten Daten von Eurostat waren im 3. Quartal 2019 7,3 \% der 15- bis 24-Jährigen (absolute Zahl: 66.000) von einer NEET-Situation betroffen (Eurostat 2020). Bei einer Abschwächung der Konjunktur ist in den nächsten Jahren ein weiterer Anstieg der Zahl der NEET-Jugendlichen zu erwarten.

Nach Bundesländern differenziert liegt Oberösterreich mit einem Prozentwert von 6,1 \% im Mittelfeld. Geringere NEET-Quoten werden für die Steiermark, Tirol und Niederösterreich ermittelt. Höhere Werte liegen für Burgenland und Wien vor (siehe Abbildung 1 sowie Tabelle A2). Statistisch signifikant ist aber nur das höhere NEET-Risiko in Wien im Vergleich zu den anderen Bundesländern mit Ausnahme von Vorarlberg (siehe Tabelle A3), wenn zweiseitige Fehlerniveaus kleiner 7,5\% (einseitig also kleiner 3,57\%) akzeptiert werden. Die Unterschiede zwischen den anderen Bundesländern sind insignifikant. Zur Analyse von Bundesländerunterschieden wären auf jeden Fall Registerdaten von Vorteil (siehe oben). Sie würden auch eine 
Analyse auf Bezirksebene ermöglichen. In Bacher et al. (2017) konnten wir nachweisen, dass numerisch kleine Unterschiede zwischen den Bundesländern Effekte auf die NEET-Rate haben.

Von den 64.000 NEET-Jugendlichen im Jahr 2018 sind $29.600(46,3 \%)$ aktiv, d. h. sie suchen zum Erhebungszeitpunkt eine Arbeitsstelle und stehen dem Arbeitsmarkt auch zur Verfügung. Vor allem ihre Zahl wird bei einer Abschwächung der Konjunktur steigen. Ein wirtschaftlicher Abschwung wird dagegen geringere Effekte auf die Zahl der inaktiven NEET-Jugendlichen ausüben, deren Anteil derzeit bei 53,7 \% liegt (absolute Zahl: 34.400). Inaktive NEET-Jugendliche sind jene Jugendliche, die nicht durch die Arbeitslosendefinition erfasst werden, also dem Arbeitsmarkt nicht zur Verfügung stehen. Nicht ausgeschlossen werden kann aber, dass sich arbeitslose NEET-Jugendliche aus Enttäuschung aus der aktiven Stellensuche zurückziehen und in den Status der inaktiven NEET-Jugendlichen wechseln. Zudem ist zu befürchten, dass bei einem wirtschaftlichen Abschwung die Verweildauer in dem aktiven und inaktiven NEET-Status steigt und es wesentlich intensiverer Anstrengung bedarf, inaktive NEET-Jugendliche zu aktivieren.

Im Bundesländervergleich auffallend ist, dass Tirol und Vorarlberg mit 61,9 \% bzw. 63,2 \% einen sehr hohen Anteil an inaktiven NEET-Jugendlichen haben. In den anderen Bundesländern streuen die Werte zwischen 53,0 \% und 56,0 \%. Die Unterschiede verfehlen aber die statistische Signifikanz. ${ }^{9}$ Es bleibt somit weiteren zukünftigen Analysen vorbehalten, ob die beobachtbaren Differenzen substanziell sind und wie sie gegebenenfalls erklärt werden können.

Statistisch betrachtet (siehe Tabelle A1) sind von NEET im Jahr 2018 häufiger ältere Jugendliche zwischen 20 und 24 Jahren, Jugendliche in Städten sowie Jugendliche mit Migrationshintergrund betroffen, wobei Jugendliche der ersten Generation ein höheres Risiko aufweisen als jene der zweiten Generation und diese wiederum ein höheres als Jugendliche ohne Migrationshintergrund. Geschlechterunterschiede sind zwar vorhanden, verfehlen aber die statistische Signifikanz.

Hauptursachen von NEET sind Erkrankungen und ein früher Schulabgang. Bei jungen Frauen kommen Betreuungsverpflichtungen hinzu. Frühe SchulabgängerInnen, also jene, die maximal über einen Pflichtschulabschluss verfügen, habe ein 10,4-mal so hohes

$9 \mathrm{Chi}_{2}=1,1 ; \mathrm{df}=2 ; \mathrm{p}=0,589$
Risiko (40,7 \% versus 3,9 \%), in eine NEET-Situation zu geraten als SchulabgängerInnen, die mindestens über einen mittleren Bildungsabschluss (Lehre oder BMS) verfügen. Im Fall einer Erkrankung ist das Risiko 6,5mal $(39,7 \% \text { vs. } 6,1 \%)^{10}$ so hoch und beim Vorhandensein von Kindern unter 6 Jahren im Haushalt 3,8-mal $(22,2 \%$ vs. $5,8 \%)$ so hoch, wobei letzterer Faktor nur bei jungen Frauen wirkt.

Die sozio-demografischen Variablen (Alter, Migrationshintergrund, Einwohnerzahl) wirken - mit einer Ausnahme - nur indirekt, wie die explorativen Pfadanalysen der Abbildungen 2 und 3 zeigen. Der Abbildung 2 ist zu entnehmen, dass weibliche NEET-Jugendliche der ersten Zuwanderergeneration mit einer größeren Wahrscheinlichkeit frühere SchulabgängerInnen sind, wobei der frühe Schulabgang auch dadurch zustande kommen kann, dass die Zuwanderung erst nach der Schulpflicht erfolgt (Bacher et al. 2014). Zuwanderinnen der ersten Generation leben auch mit einer größeren Wahrscheinlichkeit in Haushalten mit einem Kind unter 6 Jahren. Den anderen soziodemografischen Variablen (Alter, EinwohnerInnenzahl) kommt mit einer Ausnahme keine direkte Wirkung zu. Einschränkend sei hier aber angemerkt, dass im MZ die soziale Herkunft der NEET-Jugendlichen (Bildung, Beruf und Einkommen der Eltern) nicht bekannt ist. Die soziale Herkunft übt aber auf den Bildungserfolg, z. B. den Besuch einer weiterführenden Schule, einen stärkeren Einfluss aus als der Migrationshintergrund (Bruneforth et al. 2012). Allerdings scheint dies nicht für den frühen Bildungsabbruch zu gelten. Hier kommt der sozialen Herkunft eine geringere Bedeutung und dem Migrationshintergrund ein stärkerer Effekt zu (Steiner et al. 2016: 193). Mit dem Migrationshintergrund wurde somit ein wichtiger sozio-demografischer Faktor erhoben, die Bedeutung der sozialen Herkunft ist vermutlich geringer, lässt sich aber wegen fehlender Information im $\mathrm{MZ}$ nicht ermitteln.

Die feststellbaren bivariaten Zusammenhänge zwischen den betrachteten sozio-demographischen Merkmalen und dem NEET-Status sind darauf zurückzuführen, dass diese Variablen mit der Zugehörigkeit zur ersten Zuwanderergeneration im Zusammenhang stehen. So leben z. B. ZuwandererInnen der ersten Generation häufiger in Städten und daher tritt in Städten ein höheres NEET-Risiko auf. Eine Ausnahme stellt die direkte Wirkung des Alters auf das NEET-Risiko

10 Wegen struktureller Nullen wird dieses Risiko vermutlich überschätzt (siehe Erklärungen zu Tabelle A1). 
Abbildung 2: NEET-Risiko von weiblichen Jugendlichen in Abhängigkeit von statistischen Risikofaktoren (Ergebnisse einer explorativen Pfadanalyse)

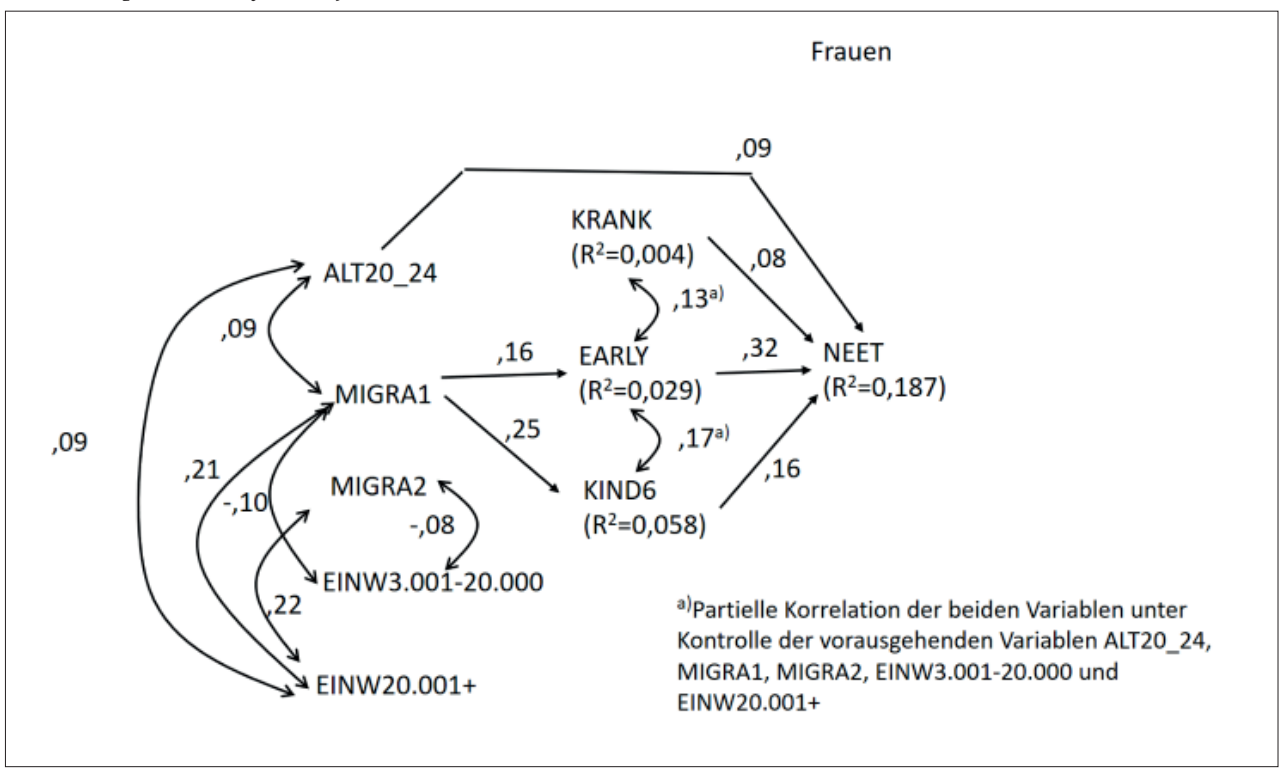

Quelle: MZ2018, gewichtete Daten, eigene Berechnungen, neff $=1.688$

Die Variablen bedeuten: ALT20_24=Alter zwischen 20 und 24 Jahren (1=ja, o=nein), MIGRA1=Zugehörigkeit zur ersten Zuwanderergeneration ( $1=\mathrm{ja}, \mathrm{o}=$ nein), MIGRA2=Zugehörigkeit zur Zweiten Migrationsgeneration ( $1=\mathrm{ja}$, o=nein), EINW3.001-20.0oo=Wohnort mit einer EinwohnerInnenzahl zwischen 3.001 und 20.000 (1=ja, o=nein), EINW20.001+=Wohnort mit einer EinwohnerInnenzahl mit mehr als 20.000 ( $1=\mathrm{ja}, \mathrm{o}=$ nein), KRANK=Erkrankung ( $1=\mathrm{ja}, \mathrm{o}=$ nein), EARLY=früher Schulabgang $(1=\mathrm{ja}$, $\mathrm{o}=$ nein), KIND6=Kinder unter 6 Jahren im Haushalt ( $1=\mathrm{ja}, \mathrm{o}=$ nein), NEET=NEET-Status $(1=\mathrm{ja}, \mathrm{o}=$ nein $)$.

Abbildung 3: NEET-Risiko von männlichen Jugendlichen in Abhängigkeit von statistischen Risikofaktoren (Ergebnisse einer explorativen Pfadanalyse)

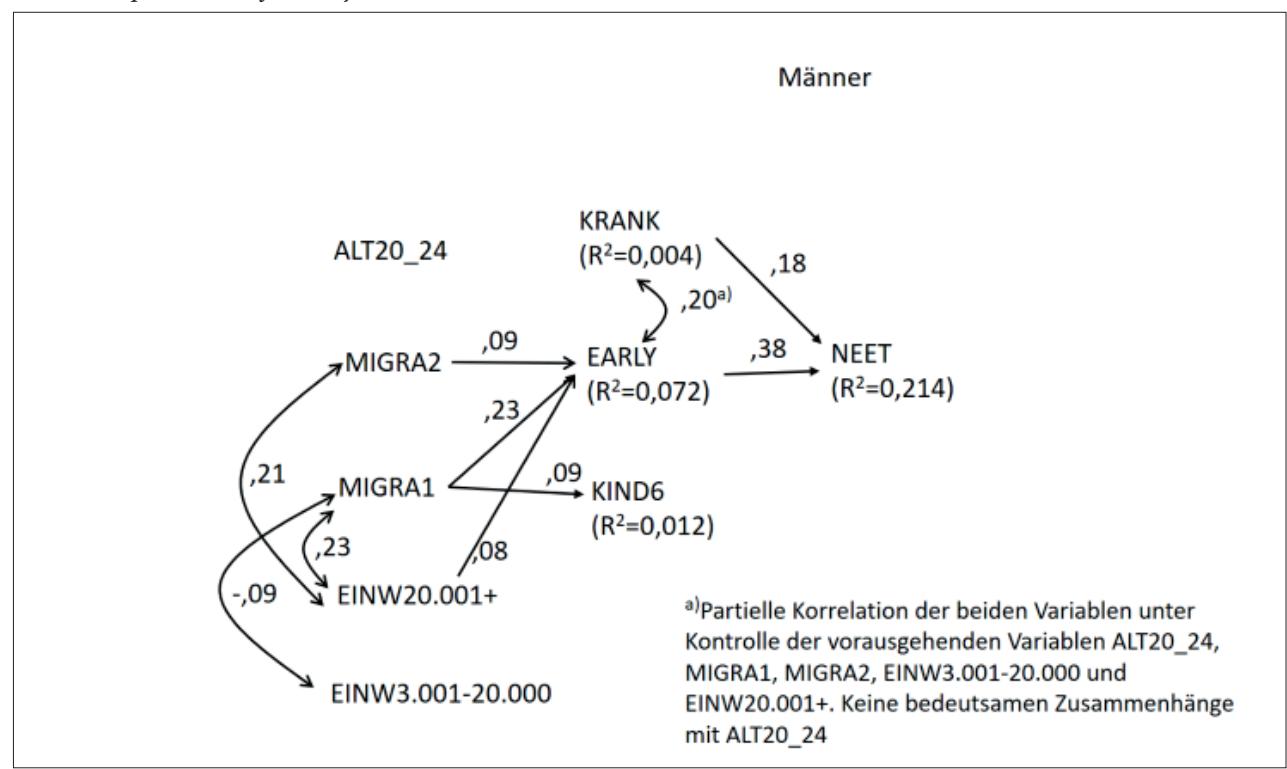

Quelle: MZ2018, gewichtete Daten, eigene Berechnungen, neff $=1.686$

Die Variablen bedeuten: siehe Abbildung 2

dar. Ceteris paribus haben 19- bis 24-Jährige junge Frauen ein höheres NEET-Risiko als die 15- bis 19-Jährigen. Ein früher Schulabgang und das Vorhandensein von mindestens einem Kind unter 6 Jahren im Haushalt wirken mit den Erkrankungen ebenfalls direkt auf das NEET-Risiko ein, wobei dem frühen Schulabgang 
Tabelle 2: Gründe von inaktiven NEET-Jugendlichen für keine Stellensuche (Spaltenprozente)

\begin{tabular}{lccc}
\hline \multicolumn{1}{c}{ Gründe für keine Stellensuche } & Männlich & Weiblich & Gesamt \\
\hline Schulische oder berufliche Ausbildung & $8,3 \%$ & $3,1 \%$ & $5,4 \%$ \\
Andere persönliche oder familiäre Umstände (Haushaltsführend) & $10,4 \%$ & $23,4 \%$ & $17,9 \%$ \\
Kinder oder pflegebedürftige Erwachsene betreuen & - & $31,3 \%$ & $17,9 \%$ \\
Krankheit oder Arbeitsunfähigkeit & $43,8 \%$ & $14,1 \%$ & $26,8 \%$ \\
Bereits eine neue Tätigkeit gefunden & $2,1 \%$ & $3,1 \%$ & $2,7 \%$ \\
Warten auf Resultate früherer Schritte zur Arbeitssuche & $4,2 \%$ & $1,6 \%$ & $2,7 \%$ \\
Rückkehr auf den alten Arbeitsplatz & $10,4 \%$ & $4,7 \%$ & $7,1 \%$ \\
Anderen Gründen & $20,8 \%$ & $18,8 \%$ & $19,6 \%$ \\
Gesamt & $100 \%$ & $100 \%$ & $100 \%$ \\
$\mathrm{n}_{\text {eff }}$ & 48 & 64 & 112 \\
\hline
\end{tabular}

Quelle: MZ2018, eigene Berechnungen

die stärkste Wirkung zukommt. Dieser steht auch im engen Zusammenhang einerseits mit Erkrankungen und andererseits mit Kindern unter 6 Jahren im Haushalt. Kausale Aussagen dahingehend, ob eine Erkrankung zu einem frühen Schulabgang oder umgekehrt ein früher Schulabgang zu Erkrankungen führt, sind nicht möglich. Gleiches gilt für Kinder unter 6 Jahren. Die Geburt eines Kindes kann einen Schulabbruch bedingen oder umgekehrt können frühe Schulabgängerinnen häufiger Mütter werden.

Die Ergebnisse der explorativen Pfadanalyse für die männlichen Jugendlichen entsprechen weitgehend jenen der weiblichen Jugendlichen mit einigen Ausnahmen. Dem Vorhandensein von Kindern unter 6 Jahren kommt kein direkter Effekt auf das NEET-Risiko zu, was darauf hinweist, dass auch unter jungen Eltern die Kinderbetreuung Frauensache ist (siehe dazu auch Tabelle 2). Im Unterschied zu den weiblichen Jugendlichen haben auch männliche Jugendliche der zweiten Zuwanderergeneration und männliche Jugendliche in Städten ein höheres Risiko eines frühen Schulabgangs. Bei den weiblichen Jugendlichen wirkt ausschließlich die Zugehörigkeit zur ersten Generation auf die Wahrscheinlichkeit eines frühen Schulabgangs ein. Nur indirekt via Korrelation wirkt das Leben in Kleinstädten. Dem Alter kommt bei den männlichen Jugendlichen nicht einmal eine nennenswerte korrelative Bedeutung $\mathrm{zu}$.

Die durchgeführten statistischen Analysen geben erste Hinweise auf mögliche Ursachen von NEET, die auch für politische Handlungsempfehlungen genutzt werden können (siehe dazu unten). Das Bild ist aber sehr unvollständig und die Prognosekraft für den
Einzelfall ist gering. Ein früher Schulabschluss beispielsweise erhöht zwar die Wahrscheinlichkeit einer NEET-Situation und daher ist es sinnvoll, die Reduktion des frühen Schulabgangs als politisches Ziel zu formulieren. Das Auftreten eines frühen Schulabgangs im Einzelfall ist aber im Regelfall eine Kumulation von Risikofaktoren bei gleichzeitigem Fehlen von Schutzfaktoren, wie qualitative Studien zeigen (Bacher et al. 2014; Lankmayer/Rigler 2016). Bei einem Jugendlichen mit einem frühen Schulabgang können z. B. fehlende Unterstützung durch Eltern, soziale Ausgrenzung durch Schulkameraden und Suchtprobleme hinzukommen. In der konkreten Arbeit mit Jugendlichen ist auf diese Individualität Bezug zu nehmen.

Auch für die Befunde in Tabelle 2 gelten die oben formulierten Einschränkungen. Tabelle 2 verdeutlicht die Heterogenität der NEET-Jugendlichen. So nennen beispielsweise 26,4 \% eine Krankheit als Grund dafür, dass sie aktuell keine Stelle suchen. Dieser Prozentsatz liegt deutlich über den in Kapitel 1 genannten 20,5 \% für psychische Beeinträchtigungen, was dadurch erklärbar ist, dass im Mikrozensus keine Unterscheidung zwischen psychischen und physischen Beeinträchtigungen möglich ist. Obwohl Tabelle 2 ein Bild der Heterogenität der NEET-Jugendlichen vermittelt, erfasst sie diese nur unzureichend. Erstens werden sehr häufig andere Gründe angeführt. Zweitens fehlen feinere Differenzierungen. Bei den Personen mit familiären Verpflichtungen z. B. ist sozialpolitisch die Unterscheidung wichtig, ob es sich um junge Mütter mit geringer schulischer Qualifikation handelt oder um bereits etwas ältere Mütter, die eine Matura als höchsten Abschluss aufweisen. Drittens ist nicht ausgeschlossen, dass einzelne Gründe auch bei 
Tabelle 3: Volkswirtschaftliche Kosten von NEET

\begin{tabular}{|c|c|c|c|}
\hline & 2018 in Euro & $\begin{array}{l}\text { Nichtgerundete Werte } \\
\text { für Österreich } 2018\end{array}$ & $\begin{array}{l}\text { Gerundete Werte für } \\
\text { Österreich } 2018\end{array}$ \\
\hline \multicolumn{4}{|l|}{ EUROFUND-Berechnung } \\
\hline $\begin{array}{l}\text { NEET-Jugendliche im Alter von } 15 \text { bis } 24 \text { Jahren, die } 6 \text { Monate oder länger } \\
\text { eine Arbeit suchen oder Nichterwerbspersonen sind }\end{array}$ & 1 & 43.548 & 43,5 Ts. \\
\hline Einkommen aus Transferzahlungen („unit public finance cost“) & 1.271 & 55.349 .508 & 55,3 Mio. \\
\hline $\begin{array}{l}\text { Entgangenes Einkommen inkl. entgangener Einnahmen von Steuern und } \\
\text { Sozialbeiträgen (.,unit resource cost“) }\end{array}$ & 16.520 & 719.412 .960 & 719,4 Mio. \\
\hline Gesamtkosten (.,unit total cost“) & 17.791 & 774.762 .468 & 774,8 Mio. \\
\hline \multicolumn{4}{|l|}{ STAATLICHER EINNAHMENENTFALL } \\
\hline Arbeitnehmerln: Sozialversicherung und Lohnsteuera) & 2.559 & 111.425 .397 & 111,4 Mio. \\
\hline Dienstgeberbeitrag (ohne DZ)a) & 4.635 & 201.854 .561 & 201,9 Mio. \\
\hline $\begin{array}{l}\text { Einnahmen aus Umsatzsteuer unter der Annahme eines Mischsteuersatzes } \\
\text { von } 16 \% \text { bei einer Sparquote von } 2 \% \text {. }\end{array}$ & 2.189 & 95.326 .572 & 95,3 Mio. \\
\hline Einnahmenverlust-Staat & 9.383 & 408.610 .884 & 408,6 Mio. \\
\hline
\end{tabular}

Quellen und Daten: Eurofund (2012), Mikrozensus 2018, eigene Berechnungen

a) Ermittelt mittels des Brutto-Netto-Rechners des Bundesministeriums für Finanzen, siehe Kapitel 2.

Nicht-NEET-Jugendlichen auftreten, es also offen ist, wie trennscharf sie für diese Gruppe sind.

\section{Volkswirtschaftliche Kosten von NEET}

Für die Schätzung der Gesamtkosten ist eine Multiplikation der für einen NEET-Jugendlichen geschätzten Kosten (siehe Kapitel 2) mit der Gesamtzahl der betroffenen Jugendlichen erforderlich. Eurofund (2012) nimmt hier eine Einschränkung vor, dass von den aktiven NEET-Jugendlichen nur jene einbezogen werden, die sechs Monate oder länger arbeitssuchend sind oder der Gruppe der Nicht-Erwerbspersonen angehören. Nimmt man diese Einschränkungen vor, reduziert sich die Zahl der Betroffenen von ca. 64.00o auf ca. 43.500 Personen (siehe Tabelle 3). Damit ergeben sich für das Jahr 2018 für Österreich wirtschaftliche Kosten in der Höhe von $€ 775$ Millionen pro Jahr, wobei die Altersgruppe im Unterschied zu Eurofund nicht geändert und mit den 15- bis 24-Jährigen gerechnet wurde.

Die hier vorgenommene Aktualisierung der volkswirtschaftlichen Kosten lässt sich in mehrfacher Hinsicht diskutieren und kritisieren. So könnte man z. B. einwenden, dass eine weitere Eingrenzung des Personenkreises, für den die Hochrechnung vorgenommen wird, sinnvoll wäre. Es könnte beispielsweise überlegt werden, NEET-Jugendliche mit familiären Betreuungspflichten auszuschließen, da sie sinnvolle, aber eben nicht bezahlte Tätigkeiten ausüben. Dabei wäre aber $\mathrm{zu}$ berücksichtigen, dass diese möglicherweise nur eine geringe Bildung haben und Bildungsmaßnahmen langfristig ihre Einkommenschancen erhöhen könnten. Allgemein ist bei einer eventuellen Einschränkung der Gruppe, für die eine Hochrechnung der volkswirtschaftlichen Kosten vorgenommen wird, zu beachten, dass sich dadurch auch die Kosten je Person ändern können. Soll sich die Kostenschätzung beispielsweise auf besonders ausgrenzungsgefährdete NEET-Jugendliche konzentrieren, sinkt zwar deren Zahl, umgekehrt steigen aber die Kosten je Person, sodass sich in der Summe derselbe Betrag ergeben kann.

Bezüglich der erfassten Kosten könnte man ferner als Kritik anführen, dass nicht das gesamte entgangene Einkommen berücksichtigt werden sollte, sondern nur die daraus entstehenden Transferleistungen an die öffentliche Hand. Um diesem Einwand Rechnung zu tragen, wurde zusätzlich der Einnahmeentfall der öffentlichen Hand berechnet. Er beträgt ca. $€ 9$ 9.400,- pro NEET-Jugendlichen. Hochgerechnet auf die Zahl von 43.50o NEET-Jugendlichen resultiert ein Einnahmeentfall der öffentlichen Hand von $€$ 408,6 Millionen.

$\mathrm{Zu}$ geringeren Werten gelangen Steiner et al. (2019) in ihren Simulationsstudien im Rahmen der Evaluierung der „AusBildung bis 18 Jahren“. Die AutorInnen untersuchen darin die langfristigen ökonomischen und budgetären Effekte, die durch eine Reduktion der frühen SchulabgängerInnen erzielt werden können (Steiner et al. 2019: 11-42). Sie beschäftigen sich also mit einer 
etwas anders gelagerten Fragestellung. Eine Senkung des frühen Schulabgangs von 18,3 \% auf 15,0 \% würde entsprechend den durchgeführten Berechnungen nach zehn Jahren zu einem zusätzlichen BIP von $€ 110$ Mio. jährlich führen. Die Reduktion des öffentlichen Budgets würde ebenfalls jährlich $€ 70$ Mio. betragen. Im Unterschied dazu führen unsere Modellrechnungen zu einem zusätzlichen BIP von $€ 774,8$ Mio. und einer Budgetreduktion von $€ 408,6$ Mio.

Die Unterschiede der beiden Schätzungen lassen sich dadurch erklären, dass Steiner et al. (2019) die Reduktion der frühen SchulabgängerInnen, also einer Gruppe, die im Unterschied zu NEET-Jugendlichen in den Arbeitsmarkt integriert sein kann, untersuchen. Unsere Berechnungen beziehen sich dagegen auf NEET-Jugendliche (genauer eine Teilgruppe davon). Des weiteren nehmen Steiner et al. (2019) an, dass Maßnahmen zur Reduktion des frühen Schulabgangs nur bei einem Teil der Jugendlichen wirken und die Quote der frühen SchulabgängerInnen insgesamt von 18,3 \% auf 15,0 \% zurückgeht, also relativ um ca. $18 \%$. Wir haben dagegen angenommen, dass alle untersuchten NEET-Jugendlichen eine Beschäftigung finden. Nimmt man vergleichbar zu der Simulationsstudie von Steiner et al. (2019) an, dass $18 \%$ der NEET-Jugendlichen eine Beschäftigung finden, so ergibt sich ein volkswirtschaftlicher Nutzen, das heißt eine Reduktion der volkswirtschaftlichen Kosten, von ca. $€ 139,5$ Mio. und eine Reduktion der öffentlichen Ausgaben um $€ 73,5$ Mio. Die Abweichungen zu den Simulationsergebnissen von Steiner et al. (2019) sind nur mehr gering. Die Unterschiede sind somit im Wesentlichen darauf zurückzuführen, welche Annahmen bezüglich der Wirksamkeit getroffen werden.

Trotz der genannten Einwände und den unterschiedlichen Rechenergebnissen vermitteln die Berechnungen eine Vorstellung darüber, dass aus volkswirtschaftlichen Gründen Maßnahmen zur Reduktion der NEET-Quote sinnvoll sind. Auch aus staatlicher Sicht sind entsprechende Maßnahmen zu empfehlen, da der Einnahmenentfall beträchtlich ist.

Hinzu kommen soziale und politische Gründe (Steiner et al. 2019; Eurofund 2012) sowie menschenrechtliche Überlegungen. In diesem Zusammenhang seien Artikel 23 und Artikel 26 der Allgemeinen Erklärung der Menschenrechte in Erinnerung gerufen.

„Artikel 23

(1) Jeder hat das Recht auf Arbeit, auffreie Berufswahl, auf gerechte und befriedigende Arbeitsbedingungen sowie auf Schutz vor Arbeitslosigkeit.

(..)
Artikel 26

(1) Jeder hat das Recht auf Bildung. Die Bildung ist unentgeltlich, zum mindesten der Grundschulunterricht und die grundlegende Bildung. Der Grundschulunterricht ist obligatorisch. Fach- und Berufsschulunterricht müssen allgemein verfügbar gemacht werden, und der Hochschulunterricht muss allen gleichermaßen entsprechend ihren Fähigkeiten offenstehen.

(2) Die Bildung muss auf die volle Entfaltung der menschlichen Persönlichkeit und auf die Stärkung der Achtung vor den Menschenrechten und Grundfreiheiten gerichtet sein. Sie muss zu Verständnis, Toleranz und Freundschaft zwischen allen Nationen und allen rassischen oder religiösen Gruppen beitragen und der Tätigkeit der Vereinten Nationen für die Wahrung des Friedens förderlich sein.

\section{(...)“(United Nations 2019)}

In der österreichischen Bundesverfassung kommt u. a. dieser Anspruch dadurch zum Ausdruck, dass als formale Bildungsziele herkunftsunabhängige Chancengleichheit und ein garantiertes Bildungsminimum, das eine umfassende Teilhabe am gesellschaftlichen, politischen und wirtschaftlichen Leben ermöglicht, verankert sind (Bruneforth et al. 2012). Auch inhaltlich wird analog zu Art. 26, Absatz 2 der Menschenrechtserklärung Bildung umfassend verstanden. Maßnahmen zur NEET-Reduktion sind daher nicht nur wirtschaftlich zu sehen, sondern vor dem Hintergrund dieses umfassenden Bildungs- und Teilhabeanspruchs.

\section{Handlungsempfehlungen für NEET-Maßnahmen}

In der Zwischenzeit liegen auf nationaler und internationaler Ebene zahlreiche Strategieüberlegungen zur Reduktion der NEET-Quote vor. In einem 2018 publizierten Bericht befasst sich die Europäische Kommission (European Commission 2018) mit Maßnahmen zur Erreichung von NEET-Jugendlichen. In Österreich stehen der Übergang und die Integration in den Arbeitsmarkt im Vordergrund (Bundesministerium für Arbeit, Soziales, Gesundheit und Konsumentenschutz 2019). Zu nennen sind hier beispielhaft das Jugendcoaching, die Einführung der Ausbildungspflicht bis 18 Jahre oder die Ausbildungsgarantie bis 25 Jahre. Eine vom Institut für Höhere Studien (IHS) und dem Österreichischen Institut für Berufsbildungsforschung (ÖIBF) durchgeführte Evaluierung der "AusBildung bis 18 Jahre“ (Steiner et al. 2019) dokumentiert und beschreibt sehr ausführlich quantitativ und qualitativ wichtige Maßnahmen für eine Erhöhung des Ausbildungsniveaus der bis 18-Jährigen, 
wie z. B. Praxis-Handelsschulen oder die überbetriebliche Ausbildung. Auch über das Jugendcoaching, um eine weitere wichtige Maßnahme $\mathrm{zu}$ nennen, liegen positive Erfahrungsberichte vor (Bundesweite Koordinierungsstelle AusBildung bis 18 (BundesKOST) 2018).

Quantitativ lässt sich in den beiden Jahren 2017 und 2018 im Vergleich zu den vorausgehenden drei Jahren (2014 bis 2016) ein Rückgang der NEET-Quote der 15bis 19-Jährigen von 5,2 \% auf 4,2 \% beobachten, ${ }^{11} \mathrm{zu}$ dem vermutlich die soeben genannten Anstrengungen beigetragen haben. $\mathrm{Ob}$ diese geringe Quote gehalten oder sogar noch weiter reduziert werden kann, wird von den politischen Weichenstellungen der Zukunft abhängen.

Für die Wirksamkeit von Maßnahmen werden in der Literatur folgende Gestaltungsprinzipien als bedeutsam erachtet (Bacher et al. 2014; Bacher et al. 2016; Dahme/Wohlfahrt 2011; European Commission 2018; Sailer/Tamesberger 2013):

- Prävention bzw. frühe Intervention. Am wirksamsten sind präventive Maßnahmen bzw. frühe Interventionen, durch die das erste Auftreten einer NEET-Situation vermieden oder durch die bei deren erstem Auftreten rasch interveniert wird. Die Reduktion des frühen Schulabgangs sowie frühe Interventionen bei Anzeichen von psychischen Belastungen oder von Kindeswohlgefährdungen sind hier die zentralen Handlungsfelder. So z. B. ergaben sich bereits bei der Geburt Hinweise auf das Vorliegen von Risikofaktoren (Pruckner/Schober 2016).

- Berücksichtigung der Heterogenität durch zielgruppenspezifische Programme. Die NEETJugendlichen stellen eine heterogene Gruppe mit unterschiedlichen Bedarfslagen dar. Auf diese unterschiedlichen Bedürfnisse ist bei der Gestaltung von Maßnahmen Rücksicht zu nehmen und zielgruppenorientiert vorzugehen. So z. B. kann es bei NEET-Jugendlichen mit psychischen Beeinträchtigungen erforderlich sein, geringfügige Arbeitsangebote von wenigen Stunden in der Woche anzubieten und zu finanzieren.

- Niedrigschwellige Angebote. Entsprechend der Heterogenität der NEET-Jugendlichen bedarf ein Teil von ihnen niedrigschwelliger und aufsuchender Angebote, um z. B. die Scheu zu überwinden, bestimmte Hilfe aufzusuchen.

- Nachhaltigkeit und Nachbetreuung. Zur Vermeidung einer erneuten Rückkehr in eine NEET-Situation ist einerseits eine gute und individuelle Vorbereitung auf ein Verlassen der NEET-Situation wichtig, zum

$11 \mathrm{Chi}^{2}=4,2 ; \mathrm{df}=1 ; \mathrm{p}=0,040$ anderen eine Nachbetreuung, wenn der Ausstieg aus NEET gelungen ist, um eine Rückkehr zu vermeiden. Maßnahmen laufen derzeit unmittelbar dann aus, wenn die NEET-Situation erfolgreich bewältigt wurde. Dies kann zu früh sein und zu einem erneuten Auftreten der NEET-Situation führen, wobei bei jedem Auftreten ein erfolgreicher Ausstieg schwieriger wird. Diese Gefahr könnte durch eine Nachbetreuung reduziert werden.

- Flexibilisierung der Altersgrenze. So z. B. ist derzeit oft mit 18 Jahren ein Wechsel von der Jugendpsychiatrie zur Erwachsenenpsychiatrie erforderlich. Damit ändert sich die Betreuungssituation maßgeblich, häufig ohne Begleitung (Plener 2019).

- Vernetzung der AkteurInnen. Erfolgreiche Unterstützung eines NEET-Jugendlichen erfordert die Zusammenarbeit unterschiedlicher AkteurInnen. Daher sind Aktivitäten erforderlich, die die Kooperation und Vernetzung der AkteurInnen fördern, die sowohl die operative Ebene (Unterstützung im konkreten Fall eines Jugendlichen) als auch die strategische Ebene (Planung von Maßnahmen) umfassen sollte.

Ein konkretes Beispiel, in dem diese Handlungsempfehlungen mit Ausnahme der Forderung nach einer frühen Intervention (Zielgruppe waren Jugendliche!) umgesetzt sind, ist das Projekt „resp@ct“von pro mente Oberösterreich (Pro Mente OÖ Jugend 2019). Das Projekt wurde im Zeitraum von 2017 bis 2019 aus Mitteln des Europäischen Sozialfonds (ESF) gefördert. Neben Angeboten für Gruppenaktivitäten, in denen die Jugendlichen fachliche Fertigkeiten erwerben bzw. nachholen konnten und positive soziale Rückmeldungen erhalten, wurde jeder/jede Jugendliche durch einen/ eine BezugsbetreuerIn begleitet. Jeder/jede im Team brachte Zusatzqualifikationen mit, sodass ein inhaltlich breites Angebot zur Verfügung gestellt werden konnte. Zusätzlich wurden externe LektorInnen eingestellt, auch ehrenamtlich Tätige konnten gewonnen werden. Der Zugang war niedrigschwellig. Durch das Coaching der Jugendlichen wurden Arbeitsbeziehungen zu unterschiedlichen NetzwerkpartnerInnen aufgebaut. Mit 2019 liefen die ESF-Förderungen aus, sodass der Betrieb von „resp@ct“ deutlich reduziert werden musste.

\section{Fazit}

Der NEET-Indikator ist zu einem festen Bestandteil der Sozialberichterstattung geworden und dient als Referenzgröße für sozial-, arbeits- und bildungspolitische Maßnahmen. Der Anteil der von NEET betroffenen Jugendlichen im Alter von 15 bis 24 Jahren betrug 
in Österreich 6,8 \% im Jahr 2018. Absolut betrachtet befanden sich ca. 64.000 Jugendliche in einer NEETSituation. Für 2019 wird ein leichter Anstieg berichtet.

Statistisch häufiger von NEET betroffen sind ältere Jugendliche, Jugendliche mit Migrationshintergrund und Jugendliche in Städten. Diese Faktoren wirken im Hintergrund. Einen direkten Einfluss auf das NEETRisiko üben der frühe Schulabgang und Erkrankungen aus, bei weiblichen Jugendlichen zusätzlich Betreuungspflichten. Die statistischen Analysen ermöglichen statistische Wahrscheinlichkeitsaussagen und können als Grundlage für politische Zielformulierungen dienen. Im konkreten Einzelfall ist es aber das Zusammenwirken mehrerer, jeweils verschiedener Risikofaktoren bei gleichzeitigem Fehlen von Schutzfaktoren, die zu einer NEET-Situation führen. Diese Heterogenität und Individualität ist bei der konkreten Arbeit mit betroffenen Jugendlichen zu beachten. Die dazu in diesem Beitrag formulierten Handlungsempfehlungen stellen Leitlinien dar.

Dass weiterhin Maßnahmen zur Reduktion der NEET-Situation erforderlich sind, ergibt sich auch aus menschenrechtlichen Aspekten sowie aus sozialen, politischen und ökonomischen Gründen. Die NEETSituation verursacht erhebliche volkswirtschaftliche Kosten und einen Einnahmeentfall der öffentlichen Hand. Dessen Größenordnung hängt von der angestrebten Wirksamkeit ab.

Hinsichtlich der NEET-Quote der 15- bis 19-Jährigen lässt sich in den vergangenen beiden Jahren eine Reduktion beobachten, was im Zusammenhang mit Maßnahmen zur „AusBildung bis $18^{\text {“ }} \mathrm{zu}$ sehen ist. Damit dieser Erfolg beibehalten und ausgebaut werden kann, ist auch in Zukunft eine aktive Arbeits- und "AusBildungspolitik“ für Jugendliche erforderlich. Angesichts der Bedeutung von Erkrankungen ist eine Verknüpfung mit Agenden der Gesundheitspolitik strategisch ratsam. Gefordert sind Bund, Länder und Gemeinden/Städte als wichtige Fördergeber. Im aktuellen Regierungsprogramm (Die neue Volkspartei und Die Grünen - Die Grüne Alternative 2020) werden auf unterschiedliche Themenbereiche verteilt einige Maßnahmen genannt, wie eine ,ausreichende Finanzierung der ÜBA“ (ebd.: 258), die „Unterstützung von jungen Menschen bei Schulabbruch in Produktionsschulen und vergleichbaren Einrichtungen“ (ebd.), die Einführung einer Bildungspflicht bis 18 Jahre (ebd.: 292-293) oder „Facharztoffensive für Fächer mit Unterversorgung und Etablierung versorgungswirksamer Strukturen (...) für die Kinder- und Jugendpsychiaterin bzw. -psychiater“ (ebd.: 268). Auch „Bildungsmöglichkeiten für asylsuchende Jugendliche nach der Pflichtschule“ (ebd.: 205) werden genannt. Es ist zu hoffen, dass diese unterschiedlichen Maßnahmen zu einer Gesamtstrategie zur Bekämpfung der Ausgrenzungsgefährdung von Jugendlichen und jungen Erwachsenen verdichtet und dabei auch Projekte und Einrichtungen gefördert werden, die den genannten Kriterien, z. B. der Niedrigschwelligkeit und der Vernetzung, genügen. 


\section{Anhang A}

Tabelle A1: NEET-Risiko in Abhängigkeit von sozio-demografischen Merkmalen 2018

\begin{tabular}{|c|c|c|c|}
\hline & Effektive Stichprobengröße neff & In \% der Gesamtgruppe & NEET-Risiko je Teilgruppe \\
\hline \multicolumn{4}{|l|}{ Alter } \\
\hline 15-19 Jahre & 1512 & 45,1 & $4,4 \%$ \\
\hline 20-24 Jahre & 1842 & 54,9 & $8,8 \%$ \\
\hline Gesamt & 3354 & 100 & $C h i^{2}=26,2 ; p=0,000$ \\
\hline \multicolumn{4}{|l|}{ Geschlecht } \\
\hline Männlich & 1686 & 50,3 & $6,6 \%$ \\
\hline Weiblich & 1668 & 49,7 & $7,1 \%$ \\
\hline Gesamt & 3354 & 100 & $C h i^{2}=0,3 ; p=0,573$ \\
\hline \multicolumn{4}{|l|}{ Migrationshintergrund } \\
\hline Nein & 2493 & 74,3 & $5,2 \%$ \\
\hline Erste Generation & 427 & 12,7 & $12,9 \%$ \\
\hline Zweite Generation & 434 & 12,9 & $10,1 \%$ \\
\hline Gesamt & 3354 & 100 & $C h i^{2}=42,3 ; p=0,000$ \\
\hline \multicolumn{4}{|c|}{ Österreichische Staatsbürgerschaft } \\
\hline Nein & 546 & 16,3 & $12,3 \%$ \\
\hline $\mathrm{Ja}$ & 2808 & 83,7 & $5,8 \%$ \\
\hline Gesamt & 3354 & 100 & $C i^{2}=30,4 ; p=0,000$ \\
\hline \multicolumn{4}{|l|}{ Gemeindegröße } \\
\hline Bis inkl. 3.000 & 877 & 26,2 & $4,9 \%$ \\
\hline 3.001 bis inkl. 20.000 & 1116 & 33,3 & $5,7 \%$ \\
\hline 20.001 und mehr & 1360 & 40,6 & $9,0 \%$ \\
\hline Gesamt & 3353 & 100 & $C h i^{2}=17,0 ; p=0,000$ \\
\hline \multicolumn{4}{|l|}{ Früher Schulabgang } \\
\hline Nein & 3091 & 92,2 & $3,9 \%$ \\
\hline $\mathrm{Ja}$ & 263 & 7,8 & $40,7 \%$ \\
\hline Gesamt & 3354 & 100 & $C h i^{2}=514,2 ; p=0,000$ \\
\hline \multicolumn{4}{|c|}{ Kind unter 6 Jahren im Haushalt } \\
\hline Nein & 3147 & 93,8 & $5,8 \%$ \\
\hline $\mathrm{Ja}$ & 207 & 6,2 & $22,2 \%$ \\
\hline Gesamt & 3354 & 100 & $C h i^{2}=82,2 ; p=0,000$ \\
\hline \multicolumn{4}{|c|}{ (Dauerhafte) Erkrankung ${ }^{a}$} \\
\hline Nein & 3281 & 97,8 & $6,1 \%$ \\
\hline $\mathrm{Ja}$ & 73 & 2,2 & $39,7 \%$ \\
\hline Gesamt & 3354 & 100 & $C h i^{2}=127,0 ; p=0,000$ \\
\hline \multicolumn{4}{|l|}{ Gesamt } \\
\hline $\mathrm{Ja}$ & 3354 & 100 & $6,8 \%$ \\
\hline
\end{tabular}

Daten: Mikrozensus 2018, eigene Berechnungen

a) Es liegen strukturelle Nullen vor. Der Gesundheitszustand wurde nicht erhoben, wenn in Vollzeit gearbeitet, eine Schule besucht oder ein Studium absolviert wird. In diesen Fällen wurde angenommen, dass keine dauerhafte Erkrankung vorliegt. Das NEET-Risiko wird damit im Falle einer Erkrankung überschätzt. 
Bacher: NEET-Jugendliche in Österreich: Problemausmaß, volkswirtschaftliche Kosten und Handlungsempfehlungen

Tabelle A2: Betroffenheit von NEET nach Bundesland

\begin{tabular}{|c|c|c|c|c|c|c|c|c|}
\hline Bundesland & nein & ja & \multicolumn{2}{|c|}{ davon } & $\begin{array}{c}\text { Gesamt } \\
(15-\text { bis 24-Jährige })^{a)}\end{array}$ & in $\%$ aller & & Jon \\
\hline Steiermark & 121875 & 6707 & 3153 & 3554 & 128582 & $5,2 \%$ & $47,0 \%$ & $53,0 \%$ \\
\hline Tirol & 79500 & 4447 & 1694 & 2753 & 83947 & $5,3 \%$ & $38,1 \%$ & $61,9 \%$ \\
\hline Kärnten & 50513 & 3210 & 1487 & 1723 & 53723 & $6,0 \%$ & $46,3 \%$ & $53,7 \%$ \\
\hline Salzburg & 55477 & 3628 & 1784 & 1844 & 59105 & $6,1 \%$ & $49,2 \%$ & $50,8 \%$ \\
\hline Oberösterreich & 149699 & 9799 & 4570 & 5229 & 159498 & $6,1 \%$ & $46,6 \%$ & $53,4 \%$ \\
\hline Wien & 189304 & 21880 & 10861 & 11019 & 211184 & $10,4 \%$ & $49,6 \%$ & $50,4 \%$ \\
\hline
\end{tabular}

Daten: Mikrozensus 2018, eigene Berechnungen

a) Ohne Präsenz- und Zivildiener

Tabelle A3: Paarweise Unterschiede zwischen den Bundesländern in der NEET-Ratea)

\begin{tabular}{|c|c|c|c|c|c|c|c|c|c|}
\hline & & Bgld. & NÖ & W & Ktn. & Stm. & OÖ. & Sbg. & $\mathrm{T}$ \\
\hline \multirow{2}{*}{ NÖ } & $\mathrm{Chi}^{2}$ & 0,448 & & & & & & & \\
\hline & $p$ & 0,504 & & & & & & & \\
\hline \multirow{2}{*}{ W } & $\mathrm{Chi}^{2}$ & 0,868 & 10,09 & & & & & & \\
\hline & $p$ & 0,351 & 0,001 & & & & & & \\
\hline \multirow{2}{*}{ Ktn. } & $\mathrm{Chi}^{2}$ & 0,267 & 0,007 & 3,789 & & & & & \\
\hline & $p$ & 0,606 & 0,935 & 0,052 & & & & & \\
\hline \multirow{2}{*}{ Stm. } & $\mathrm{Chi}^{2}$ & 0,649 & 0,065 & 9,680 & 0,070 & & & & \\
\hline & $p$ & 0,420 & 0,799 & 0,002 & 0,792 & & & & \\
\hline \multirow{2}{*}{ ○Ö } & $\mathrm{Chi}^{2}$ & 0,188 & 0,166 & 7,323 & 0,041 & 0,393 & & & \\
\hline & $p$ & 0,665 & 0,684 & 0,007 & 0,840 & 0,531 & & & \\
\hline \multirow{2}{*}{ Sbg. } & $\mathrm{Chi}^{2}$ & 0,146 & 0,091 & 3,405 & 0,029 & 0,233 & 0 & & \\
\hline & $p$ & 0,702 & 0,763 & 0,065 & 0,864 & 0,629 & 0 & & \\
\hline \multirow{2}{*}{$\mathrm{T}$} & $\mathrm{Chi}^{2}$ & 0,521 & 0,026 & 6,648 & 0,039 & 0,004 & 0,237 & 0,155 & \\
\hline & $p$ & 0,471 & 0,872 & 0,010 & 0,844 & 0,453 & 0,626 & 0,693 & \\
\hline \multirow{2}{*}{ Vbg. } & $\mathrm{Chi}^{2}$ & 0,005 & 0,490 & 1,567 & 0,254 & 0,731 & 0,176 & 0,125 & 0,557 \\
\hline & $p$ & 0,943 & 0,484 & 0,212 & 0,614 & 0,393 & 0,675 & 0,724 & 0,456 \\
\hline
\end{tabular}

Daten: Mikrozensus 2018, eigene Berechnungen, keine Korrektur für Mehrfachtestung

a) Ohne Präsenz- und Zivildiener

Bgld.=Burgenland, NÖ=Niederösterreich, W=Wien, Ktn.=Kärnten, Stm.=Steiermark, OÖ=Oberösterreich, Sbg.=Salzburg, T=Tirol, Vbg.=Vorarlberg 


\section{Literaturverzeichnis}

Bacher, J. (2009): Analyse komplexer Stichproben. In: Weichbold, M./Bacher, J./Wolf C. (Hg.): Umfrageforschung. Herausforderungen und Grenzen. Wiesbaden: VS Verlag für Sozialwissenschaften / GWV Fachverlage GmbH Wiesbaden 253-273.

Bacher, J./Braun, J./Burtscher-Mathis, S./Dlabaja, C./Lankmayer, T./Leitgöb, H./Stadlmayr, M./Tamesberger, D. (2014): Unterstützung der arbeitsmarktpolitischen Zielgruppe „NEET“. Wien: ÖGB Verlag (Sozialpolitische Studienreihe, 17).

Bacher, J./Koblbauer, C./Leitgöb, H./Tamesberger, D. (2017): Small differences matter: how regional distinctions in educational and labour market policy account for heterogeneity in NEET rates. Journal of Labour Market Research, 51 (1), 1-20, 29. DOI: 10.1186/S12651-017-0232-6.

Bacher, J./Koblbauer, C./Lankmayer, T./Pruckner, G./Rigler, S./Schober, T./Tamesberger, D. (Hg.) (2016): Psychische und physische Gesundheitsbeeinträchtigungen im Jugendalter. Wien: Studie im Auftrag des Bundesministeriums für Arbeit, Soziales und Konsumentenschutz (BMASK). Online: https://www.ibe.co.at/de/forschung/forschungsprojekt/forschungsprojekt.neet-studie-psychische-undphysische-gesundheitsbelastungen-im-jugendalter. html?xtxsearchselecthit $=1$ [10.10.2019].

Bacher, J./Tamesberger, D. (2011): Junge Menschen ohne (Berufs-)Ausbildung. Ausmaß und Problemskizze anhand unterschiedlicher Sozialindikatoren. WISO 34 (4), 95-109.

Bălan, M. (2015): Methods to Estimate the Structure and Size of the "Neet" Youth. Procedia Economics and Finance, 32, 119-124. DOI: 10.1016/S2212-5671(15)01372-6.

Baumgartner, I./Moser, C./Zucha, V. (2017): Erläuterungen zum Mikrozensus 2018. Wien: Statistik Austria.

Benchimol, E.I./Smeeth, L./Guttmann, A./Harron, K./Hemkens, L./Moher, D./Petersen, I./Sörensen, H. T./Elmi, E./ Langanc, S. M. (2016): Das RECORD-Statement zum Berichten von Beobachtungsstudien, die routinemäßig gesammelte Gesundheitsdaten verwenden. Zeitschrift für Evidenz, Fortbildung und Qualität im Gesundheitswesen, 115-116, 33-48. DOI: 10.1016/j.zefq.2016.07.010.

Bonner, A. (Hg.) (2017): Social Determinants of Health. An Interdisciplinary Approach to social Ineqaultiy. Bristol: Policy Press.

Bortz, J. (1999): Statistik für Sozialwissenschaftler. Berlin, Heidelberg, New York: Springer-Verlag.

Bruneforth, M./Weber, C./Bacher, J. (2012): Chancengleichheit und garantiertes Bildungsminimum in Österreich. In: Herzog-Punzenberger B. (Hg.): Nationaler Bildungsbericht Band 2: Fokussierte Analysen bildungspolitischer Schwerpunktthemen. Graz: Leykam, 189-228.

Bundesministerium Arbeit, Soziales, Gesundheit und Konsumentenschutz (Hg.) (2019): Jugendliche und junge Erwachsene auf dem Arbeitsmarkt. Wien: BMASGK. Online: https://www.sozialministerium.at/Themen/Arbeit/
Arbeitsmarkt/Arbeitsmarktfoerderungen/Jugendlicheund-junge-Erwachsene.html [17.03.2020].

Bundesweite Koordinierungsstelle AusBildung bis 18 (BundesKOST) (2018): Jugendcoaching 2017. Wien: BundesKOST. Online: https://www.bundeskost.at/wp-content/ uploads/2018/05/Jugendcoaching-Jahresbericht-2017. pdf [20.02.2020].

Coles, B./Hutton, S./Bradshaw, J./Craig, G./Godfrey, C./Johnson, J. (2002): Literature Review of the Costs of Being "Not in Education, Employment or Training" at Age 1618: Social Policy Research Unit, University of York and University of Hall. Online: https://www.york.ac.uk/inst/ spru/pubs/pdf/RR347.pdf [17.03.2020].

Crespo Cuaresma, J./König, T./Oberhofer, H./Schwarz, G./ Strassnig, M. (2020): Plattform "Registerforschung". Wien. Online: https://www.registerforschung.at/\#top [05.03.2020].

Dahme, H./Wohlfahrt, N. (Hg.) (2011): Handbuch Kommunale Sozialpolitik. 1. Aufl. Wiesbaden: VS Verlag für Sozialwissenschaften / Springer Fachmedien Wiesbaden GmbH Wiesbaden.

Die neue Volkspartei; Die Grünen - Die Grüne Alternative (2020): Aus Verantwortung für Österreich. Regierungsprogramm 2020-2024. Wien. Online: https:// www.dieneuevolkspartei.at/Download/Regierungsprogramm_2020.pdf [20.02.2020].

Ederer, S. (2017): Österreich 2025 - Einkommensverteilung und privater Konsum in Österreich. WIFO-Monatsberichte, 90 (1), 67-81. Online: https://www.wifo. ac.at/jart/prj3/wifo/resources/person_dokument/ person_dokument.jart?publikationsid $=59263 \& \mathrm{mime}$ type $=$ application/pdf [03.03.2020].

Eurofund (2012): NEETs - Young people not in employment, education or training: Characteristics, costs and policy responses in Europe. Luxembourg: Publications Office of the European Union.

European Commission (2018): Effective outreach to NEETs. Experience from the ground. Luxembourg: Publications Office of the European Union.

Eurostat (2019): Statistics on young people neither in employment nor in education or training. Luxembourg: Eurostat. Online: https://ec.europa.eu/eurostat/statisticsexplained/index.php/Statistics_on_young_people_neither_in_employment_nor_in_education_or_training [17.03.2020].

Eurostat (2020): Young people aged 15-24 neither in employment nor in education and training (NEET), by sex - quarterly data. Brüssel: Eurostat. Online: https://appsso.eurostat.ec.europa.eu/nui/show. do?query=BOOKMARK_DS-857688_QID_7E39EoB7_ UID_-3F171EBo\&layout=TIME,C,X,o;GEO,L,Y,o;UNI T,L,Z,o;SEX,L,Z,1;S_ADJ,L,Z,2;INDICATORS,C,Z,3;\& zSelection=DS-857688S_ADJ,SA;DS-857688SEX,T;DS857688UNIT,PC_POP;DS-857688INDICATORS,OBS_ F L A G ; \& r a n k N a m e $1=\mathrm{UN} \mathrm{I} \mathrm{T}_{-}{ }^{1}{ }_{-}{ }^{2}{ }_{-}-$ $1_{-} 2 \& \mathrm{rankN}$ a me $2=$ IN D ICATORS ${ }_{-}{ }_{-}{ }_{2}{ }_{-}-$ $1 \_2 \&$ rankName $3=S E X \_1 \_2 \_-1 \_2 \&$ rankName $4=S$ - 
ADJ_1_2_-1_2\&rankName5=TIME_1_O_O_o\&rank Name6=GEO_1_2_o_1\&ppcRK=FIRST\&ppcSO=A $\mathrm{SC} \&$ sortC $=$ ASC_-1_FIRST $\& \mathrm{rStp}=\& \mathrm{cStp}=\& \mathrm{rDCh}=$ $\& \mathrm{cDCh}=\& \mathrm{rDM}=$ true $\& \mathrm{cDM}=$ true \&footnes $=$ false \&e mpty $=$ false \&wai $=$ false\&time_mode $=$ NONE\&time most_recent $=$ false $\&$ lang $=\mathrm{EN} \& \mathrm{cfo}=\% 23 \% 23 \% 23 \%$ 2C\%23\%23\%23.\%23\%23\%23 [20.02.2020].

Gracey, S./Kelly, S. (2010): Changing the NEET mindset. Achieving more effective transitions between education and work. London: LSN.

Haslinger, A./Kytir, J. (2006): Stichprobendesign, Stichprobenziehung und Hochrechnung des Mikrozensus ab 2004. Statistische Nachrichten, 61 (6), 510-519.

Holm, K. (1977): Lineare multiple Regression und Pfadanalyse. In: Holm K. (Hg.): Die Befragung, 5. München: Francke Verlag, 7-102.

Lankmayer, T./Rigler, S. (2016): Risikofaktoren und Ressourcen - Ergebnisse der Reanalyse der qualitativen Interviews mit NEET-Jugendlichen. In: Bacher, J./Koblbauer, C./Lankmayer, T./Pruckner, G./ Rigler, S./Schober, T./ Tamesberger D. (Hg.): Psychische und physische Gesundheitsbeeinträchtigungen im Jugendalter. Wien: Studie im Auftrag des Bundesministeriums für Arbeit, Soziales und Konsumentenschutz (BMASK), 87-113.

Lefering, R. (2016): Registerdaten zur Nutzenbewertung Beispiel TraumaRegister DGU $\left(^{\circ}\right)$. Zeitschrift fur Evidenz, Fortbildung und Qualitat im Gesundheitswesen, 112 Suppl 1, S11-S15. DOI: 10.1016/j.zefq.2016.04.009.

Litschel, V. (2019): Zielgruppenanalyse: NEETs. In: Steiner, M./Pessl, G./Leitner, A./Davoine, T./Forstner, S./Juen, I./Köpping, M./Sticker, A./Litschel, V./Löffler, R./Petanovitsch A. (Hg.): AusBildung bis 18. Wissenschaftliche Begleitung der Implementierung und Umsetzung des Ausbildungspflichtgesetzes. Wien: IHS, 179-187.

O'Reilly, J./Leschke, J./Ortlieb, R./Seeleib-Kaiser, M./Villa, P. (Hg.) (2019): Youth labor in transition. Inequalities, mobility, and policies in Europe. New York, NY, United States of America: Oxford University Press (International policy exchange series).

Plener, P. (2019): Erwachsen werden: Kinder- und Jugendpsychiatrie in der Adoleszenz. Jugendfachtagung 2019. Linz: Pro Mente OÖ. Online: https://www.promentejugend. at/anmeldung-jugendfachtagung/[10.10.2019].

Pro Mente OÖ Jugend (2019): resp@ct. Linz: Pro Mente OÖ. Online: https://www.promentejugend.at/angebote/niederschwellige-angebote/resp-ct/ [17.03.2020].

Pruckner, G./Schober, T. (2016): Analyse von administrativen Daten - Arbeitsmarktintegration und Gesundheit Jugendlicher. In: Bacher, J./Koblbauer, C./Lankmayer, T./Pruckner, G./ Rigler, S./Schober, T./Tamesberger D. (Hg.): Psychische und physische Gesundheitsbeeinträchtigungen im Jugendalter. Wien: Studie im Auftrag des Bundesministeriums für Arbeit, Soziales und Konsumentenschutz (BMASK), 58-86.

Sailer, B./Tamesberger, D. (2013): NEET-Jugendliche und politische Partizipation. Der Beitrag der sozialräumlichen
Jugendarbeit zur Steigerung der politischen Partizipation. Momentum Quarterly, 2 (4), 168-230.

Statistik Austria (2019a): Jährliche Personeneinkommen. Wien: Statistik Austria. Online: http://www.statistik.at/ web_de/statistiken/menschen_und_gesellschaft/soziales/personen-einkommen/jaehrliche_personen_einkommen/index.html [10.10.2019].

Statistik Austria (2019b): Nichterwerbstätige Jugendliche (NEETs). Wien: Statistik Austria. Online: https://www. statistik.at/web_de/statistiken/menschen_und_gesellschaft/bildung/bildungsindikatoren/nichterwerbstaetige_jugendliche/index.html [09.10.2019].

Statistik Austria (2019c): Statistik der Umsatzsteuer 2016. Wien: Statistik Austria. Online: http://www.statistik.at/ web_de/services/publikationen/19/index.html?includeP age $=$ detailedView\&sectionName $=\% \mathrm{C}_{3} \% 96 \mathrm{ffentliche}+\mathrm{Fi}$ nanzen $\% 2 \mathrm{C}+$ Steuern\&pubId $=575$ [03.03.2020].

Steiner, M./Pessl, G./Bruneforth, M. (2016): Früher Bildungsabbruch - Neue Erkenntnisse zu Ausmaß und Ursachen. In: Bruneforth, M./Eder, F./Krainer, K./Schreiner, C./ Seel A./Spiel, C. (Hg.): Nationaler Bildungsbericht Österreich 2015. Band 2: Fokussierte Analysen bildungspolitischer Schwerpunktthemen. Graz: Leykam, 175-220.

Steiner, M./Pessl, G./Leitner, A./Davoine, T./Forstner, S./ Juen, I./Köpping, M./Sticker, A./Litschel, V./Löffler, R./ Petanovitsch A. (Hg.) (2019): AusBildung bis 18. Wissenschaftliche Begleitung der Implementierung und Umsetzung des Ausbildungspflichtgesetzes. IHS. Wien: IHS. Online: https://irihs.ihs.ac.at/id/eprint/5174/ [17.03.2020].

United Nations (2019): Allgemeine Erklärung der Menschenrechte. Bonn: Regionales Informationszentrum der Vereinten Nationen für Westeuropa (UNRIC). Online: https://www.unric.org/de/menschenrechte/16 [10.10.2019]. 